\title{
Rapid conversion of the ester prodrug abiraterone acetate results in intestinal supersaturation and enhanced absorption of abiraterone: in vitro, rat in situ and human in vivo studies.
}

Jef Stappaerts ${ }^{a}$, Sophie Geboers ${ }^{a}$, Danny Riethorst ${ }^{a}$, Jan Snoeys ${ }^{b}$, Joachim Brouwers ${ }^{a}$, Jan Tack ${ }^{c}$, Pieter Annaert ${ }^{\mathrm{a}}$ and Patrick Augustijns ${ }^{\mathrm{a}}$

a Drug Delivery and Disposition, KU Leuven Department of Pharmaceutical and Pharmacological Sciences, Leuven, Belgium

b Pharmacokinetics, Dynamics and Metabolism, Janssen Research and Development, Beerse, Belgium

${ }^{\mathbf{c}}$ Department of Gastroenterology, University Hospitals Leuven, Belgium

Corresponding author:

Patrick Augustijns

Drug Delivery and Disposition, KU Leuven Department of Pharmaceutical and Pharmacological Sciences

Gasthuisberg O\&N 2 - Herestraat 49 box 921 - 3000 Leuven - Belgium tel: +32-16-330301 - fax: +32-16-330305

e-mail: patrick.augustijns@pharm.kuleuven.be 


\section{$\underline{\text { Abstract }}$}

The aim of this study was to evaluate the intestinal disposition of abiraterone acetate, an ester prodrug of the anticancer agent abiraterone. Stability of the prodrug and solubility and dissolution characteristics of both abiraterone and abiraterone acetate were monitored in vitro. Moreover, the in vivo intraluminal concentrations of abiraterone and abiraterone acetate upon intake of one tablet of $250 \mathrm{mg}$ abiraterone acetate were assessed in healthy volunteers. The intestinal absorption resulting from the intraluminal behavior of the ester prodrug was determined using the rat in situ intestinal perfusion technique with mesenteric blood sampling. Simulated and aspirated human intestinal fluids of the fasted state were used as solvent systems.

Upon incubation of abiraterone acetate in human intestinal fluids in vitro, rapid hydrolysis of the prodrug was observed, generating abiraterone concentrations largely exceeding the apparent solubility of abiraterone, suggesting the existence of intestinal supersaturation. These findings were confirmed in vivo, by intraluminal sampling of duodenal fluids upon oral intake of an abiraterone acetate tablet by healthy volunteers

Rat in situ intestinal perfusion experiments performed with suspensions of abiraterone and abiraterone acetate in human intestinal fluids of the fasted state revealed significantly higher flux values upon perfusion with the prodrug than with abiraterone. Moreover, rat in situ intestinal perfusion with abiraterone acetate suspensions in simulated fluids of the fasted state in presence or absence of esterases demonstrated that increased hydrolytic activity of the perfusion medium was beneficial to the intestinal absorption of abiraterone.

In conclusion, the rapid hydrolysis of abiraterone acetate in the intraluminal environment appears to result in fast and extensive generation of abiraterone supersaturation, creating a strong driving force for abiraterone absorption. 
Keywords: ester prodrug; intestinal perfusion; human intestinal fluids, intraluminal supersaturation, abiraterone, intraluminal degradation 


\section{$\underline{\text { Introduction }}$}

Hydrolytic enzymes including esterases are involved in the hydrolysis of a significant number of marketed prodrugs. Being ubiquitously present in the enterocytes, they pose an important limitation in the absorption of drugs carrying an ester bond [1]. In addition to intracellular hydrolysis, several studies report on the instability of ester prodrugs in human intestinal fluids [2-5]. In view of the labile nature of ester prodrugs, assessment of stability in intestinal fluids is crucial. Chemical and enzymatic intraluminal stability are often very important for the prodrug approach to be successful. Therefore, evaluating the stability of a prodrug in biorelevant fluids is a crucial step in prodrug development.

Borde et al. proposed the use of dog intestinal fluids as an alternative to human intestinal fluids [2]. In the same study, the use of fasted state simulated intestinal fluids supplemented with pancreatin was also evaluated as a solvent system to determine ester prodrug stability; however, both in dog intestinal fluids and in the simulated fluids, degradation was found to be slower as compared to degradation in human intestinal fluids.

The anticancer agent abiraterone is commercialized as abiraterone acetate $\left(\mathrm{Zytiga}^{\circledR}\right)$. The permeability of abiraterone acetate across Caco- 2 monolayers is low and similar to the permeability of abiraterone. Moreover, poor aqueous solubility of the prodrug results in a BCS 4 classification of abiraterone acetate [7]. In accordance with these biopharmaceutical properties, the oral bioavailability of abiraterone upon intake of abiraterone acetate is low. As an up to 10-fold increase in abiraterone bioavailability was observed when administered with food, the systemic exposure upon oral intake should be lower than 10\% [6]. Notwithstanding the significant positive effect of food on oral bioavailability, the ZYTIGA ${ }^{\circledR}$ label states that abiraterone acetate must be taken on an empty stomach with no food consumed for at least two hours before the dose and for at least one hour after the dose in order to reduce variability in absorption. The recommended dosing is $1000 \mathrm{mg}$ once daily, and more than half of this 
dose was demonstrated to be excreted unchanged via the faeces. Moreover, abiraterone, excreted via the faeces accounted for an additional $22 \%$ of the dose, administered in this mass balance study [8].

The aim of this research was to gain insight into the mechanisms underlying the intestinal absorption of abiraterone. Solubility and stability experiments were performed in biorelevant fluids, including simulated intestinal fluids (FaSSIF) and aspirated human intestinal fluids (FaHIF) of the fasted state. Moreover, the in vivo intraluminal behavior of abiraterone acetate upon oral intake was studied in healthy volunteers. To gauge the repercussion of the intraluminal behavior of the ester prodrug on the intestinal absorption, the rat in situ intestinal perfusion technique with mesenteric blood sampling was selected as an appropriate absorption model.

\section{Materials and methods}

\section{$\underline{\text { Chemicals }}$}

Abiraterone, abiraterone acetate and their deuterated counterparts abiraterone-d4 and abiraterone acetate-d4 were kindly provided by Janssen Research \& Development (Beerse, Belgium). VWR International (Leuven, Belgium) supplied methanol. Methyl tert-butyl ether (MTBE) was purchased from Merck (Overijse, Belgium). Esterase from porcine liver was obtained from Sigma Aldrich (St. Louis, MO). Acetonitrile and dimethylsulfoxide (DMSO) were purchased from Acros Organics (Geel, Belgium). Biorelevant (Surrey, UK) supplied simulated intestinal fluid (SIF) powder. Ketamine (Anesketin) and xylazin (Xyl-M 2\%) were obtained from Eurovet (Heusden, Belgium) and VMD (Arendonk, Belgium), respectively. Stock solutions were prepared in DMSO. Water was purified with a Maxima system (Elga Ltd., High Wycombe Bucks, UK). 


\section{$\underline{\text { Media }}$}

Fasted state simulated intestinal fluids (FaSSIF) were made according to the manufacturer's protocol. Briefly, FaSSIF were prepared by dissolving SIF powder $(\mathbf{2 . 2 4} \mathbf{~ m g} / \mathbf{m l})$ in a blank FaSSIF phosphate buffer. Fasted state human intestinal fluids (FaHIF), used as a solvent system for the solubility measurements, the dissolution tests and the rat in situ intestinal perfusion experiments were collected from the duodenum of healthy volunteers in the fasted state (13 volunteers) according to the method described by Bevernage et al. (2011) [9]. The human intestinal fluids were collected every $15 \mathrm{~min}$ for up to $120 \mathrm{~min}$ from the duodenum (D2-D3) after the intake of $250 \mathrm{~mL}$ of water. One pooled sample was prepared by combining the aspirates from all volunteers. The pooled HIF were stored at $-30^{\circ} \mathrm{C}$ until further use. Approval for the collection of FaHIF from volunteers was granted by the University Hospital Medical Ethics Commission of the KU Leuven.

For the dissolution studies and the in situ intestinal perfusion experiments, the $\mathrm{pH}$ of FaSSIF was increased to 7.4, to better reflect the $\mathrm{pH}$ of the FaHIF pool.

\section{$\underline{\text { Solubility Measurements }}$}

The apparent solubility of abiraterone and abiraterone acetate was determined in FaSSIF and in FaHIF using the shake flask method. All solubility experiments were performed in triplicate. Approximately $0.3 \mathrm{mg}$ of abiraterone or abiraterone acetate (crystalline powder) was added to microcentrifuge tubes containing $0.3 \mathrm{~mL}$ of the above mentioned media and placed in a prewarmed shaking incubator $\left[37^{\circ} \mathrm{C}\right.$ at $175 \mathrm{rpm}(\mathrm{KS} 4000 \mathrm{i}$ control incubator from Ika (Staufen, Germany))] for $24 \mathrm{~h}$. In an effort to determine the time dependent changes in apparent solubility, samples were taken at $1 \mathrm{~h}, 2 \mathrm{~h}$ and $5 \mathrm{~h}$ in addition to the $24 \mathrm{~h}$ time point. The solid phase was separated from the dissolved part using centrifugation (15 min, 20.817g at $37^{\circ} \mathrm{C}$ ). The supernatant was immediately diluted $1 / 100$ in $\mathrm{MeOH}: \mathrm{H}_{2} \mathrm{O}$ (50:50). Before 
analysis, the samples were further diluted to $1 / 1000$ in $\mathrm{MeOH}: \mathrm{H}_{2} \mathrm{O}(50: 50)$ containing both abiraterone-d4 and abiraterone acetate- $\mathrm{d} 4$, used as internal standards at a final concentration of $5 \mathrm{nM}$.

\section{$\underline{\text { Dissolution tests }}$}

Dissolution characteristics and supersaturation potential were investigated by adding $2 \mathrm{mg}$ of abiraterone acetate or abiraterone to $2 \mathrm{ml}$ of FaSSIF supplemented with esterases $(20 \mathrm{U} / \mathrm{ml})$ or to $2 \mathrm{ml}$ of FaHIF. The experiments were performed in stirred medium maintained at $37^{\circ} \mathrm{C}$. After adding the abiraterone acetate powder to the test medium, samples of $100 \mu 1$ were taken at 5, 20, 40, 60 and $120 \mathrm{~min}$ and centrifuged $\left(10 \mathrm{~min}, 21000 \mathrm{~g}, 37^{\circ} \mathrm{C}\right)$. The supernatant was immediately diluted 1/100 in $\mathrm{MeOH}: \mathrm{H}_{2} \mathrm{O}$ (50:50). Before analysis, the samples were further diluted to 1/1000 in $\mathrm{MeOH}: \mathrm{H}_{2} \mathrm{O}$ (50:50) containing both abiraterone-d4 and abiraterone acetate-d4 used as internal standards at a final concentration of $5 \mathrm{nM}$.

\section{$\underline{\text { Animals }}$}

Purpose-bred, male Sprague-Dawley rats (Janvier, Le Genest Saint-Isle, France) were used for in situ intestinal perfusion experiments. The rats were housed according to the Belgian and European laws, guidelines and policies for animal experiments, housing and care in the Central Animal Facilities of the university. Approval for this project was granted by the Institutional Ethical Committee for Animal Experimentation.

\section{$\underline{\text { In situ intestinal perfusion }}$}

Rats of approximately $350 \mathrm{~g}$ were anaesthetized using a mixture of ketamin $(87.5 \mathrm{mg} / \mathrm{kg})$ and xylazin $(0.875 \mathrm{mg} / \mathrm{kg})$. The right jugular vein was cannulated with a heparinized $(50 \mathrm{IU} / \mathrm{ml})$ polyethylene cannula (o.d. 1.02mm; Portex, Kent, UK) for blood supply from donor rats 
during the perfusion experiment. A laparotomy was performed and a distal segment of the small intestine was isolated by inserting two glass cannulas (o.d. $4 \mathrm{~mm}$, i.d. $3 \mathrm{~mm}$ ) at the proximal and distal end of the segment. A mean intestinal segment radius of $0.2 \mathrm{~cm}$ was used in further calculations. Polyethylene tubing was connected to the inlet cannula and a perfusion pump (Minipuls3, Gilson, Middleton, USA) was placed between the perfusate reservoir and the inlet cannula. After removal of the intestinal content, the mesenteric vein draining the isolated part of the intestinal segment was cannulated using the top end $(1 \mathrm{~cm})$ of a catheter (Insyte-W ${ }^{\circledR} 0.7$ x $19 \mathrm{~mm}$; Beckton Dickinson, Salt Lake City, Utah) and the intestinal segment was preincubated with FaSSIF for 30 minutes at a perfusion flow rate of $1 \mathrm{ml} / \mathrm{min}$. The cannula was secured with a knot and connected to a piece of silastic tubing (o.d. $1.19 \mathrm{~mm}$, i.d. $0.64 \mathrm{~mm}$; Helix Medical, USA) for blood collection. During this $30 \mathrm{~min}$ preincubation period, a test tube containing a suspension of $3 \mathrm{mg}$ of abiraterone or abiraterone acetate in $6 \mathrm{ml}$ of FaSSIF, FaSSIF supplemented with esterase $(20 \mathrm{U} / \mathrm{ml})$ or FaHIF was placed in a water bath at $37^{\circ} \mathrm{C}$. Magnetic stirrer bars provided mixing of the medium (400 rpm). After $30 \mathrm{~min}$, the resulting suspension was used as perfusion medium. All in situ experiments were performed in the closed loop set-up to avoid excessive use of FaHIF and compound.

The perfusion flow rate for all in situ experiments amounted to $1 \mathrm{ml} / \mathrm{min}$. The blood from the mesenteric vein was continuously collected over 5-min intervals and the blood samples were immediately stored at $-30^{\circ} \mathrm{C}$ prior to analysis. Donor blood was supplied via the jugular vein at a rate of $0.3 \mathrm{ml} / \mathrm{min}$ using a syringe pump (Pilot A2, Fresenius Vial, Grenoble, France) to maintain hemodynamic conditions. To verify the donor concentration, perfusate samples were taken every $10 \mathrm{~min}$ followed by a centrifugation step $\left(5 \mathrm{~min}, 20817 \mathrm{~g}, 37^{\circ} \mathrm{C}\right)$. The supernatant was diluted 1/100 with methanol:water $(50: 50 \mathrm{v} / \mathrm{v})$. Before analysis, the samples were further diluted to 1/1000 in $\mathrm{MeOH}: \mathrm{H}_{2} \mathrm{O}$ (50:50) containing both abiraterone-d4 and abiraterone acetate-d4 as internal standards at a final concentration of $5 \mathrm{nM}$. 


\section{$\underline{\text { In vivo studies }}$}

To evaluate the intraluminal behavior of abiraterone acetate in vivo, duodenal concentrations of abiraterone acetate and abiraterone were monitored in three healthy male volunteers (aged between 22 and 24 years). The procedure followed the tenets of the Declaration of Helsinki and was approved by the Committee of Medical Ethics of the University Hospitals Leuven, Belgium (ML9149) and the Federal Agency for Medicines and Health Products (EudraCT number 2013-005594-51). All volunteers provided written informed consent to participate in this study. After a fasting period of $12 \mathrm{~h}$, a double-lumen polyvinyl catheter [Salem Sump Tube $14 \mathrm{Ch}$ (external diameter $4.7 \mathrm{~mm}$ ), Covidien, Dublin, Ireland] was introduced via the nose and positioned into the duodenum (D2/D3); positioning was checked by fluoroscopy. A tablet of $250 \mathrm{mg}$ abiraterone acetate $\left(\mathrm{ZYTIGA}^{\circledR}\right.$, Janssen-Cilag International NV) was administered orally with $250 \mathrm{ml}$ of water to the volunteers.

During the experiment, volunteers were sitting in an upright position. Samples were taken every ten minutes in the first hour, and afterwards every fifteen minutes for up to four hours. The sampling volume was kept as small as possible $(<3 \mathrm{ml})$. The $\mathrm{pH}$ of the intestinal fluids was measured immediately after aspiration (Hamilton Knick Portamess Bonaduz, Switzerland). To determine the intraluminal concentrations of abiraterone and abiraterone acetate, $300 \mu \mathrm{l}$ of the aspirated sample was immediately centrifuged ( $5 \mathrm{~min}, 20817 \mathrm{~g}, \mathrm{RT}$ ). The supernatant was diluted 1/100 in $\mathrm{MeOH}: \mathrm{H}_{2} \mathrm{O}$ (50:50) containing both abiraterone-d4 and abiraterone acetate-d4 as internal standards at a final concentration of $5 \mathrm{nM}$. Additionally, 100 $\mu 1$ of the aspirated sample was diluted 1/100 in $\mathrm{MeOH}: \mathrm{H}_{2} \mathrm{O}$ (50:50) without a centrifugation step, to estimate the total amount of abiraterone and abiraterone acetate present in the intestinal fluids. Aspirated fluids and diluted samples were stored on ice during the in vivo experiments. Afterwards, $300 \mu \mathrm{l}$ of the original aspirated sample was used to determine the 
apparent solubility of abiraterone in the different intestinal samples. Solubility measurements were performed as described above ( $0.3 \mathrm{mg}$ of abiraterone in $300 \mu \mathrm{l}$ of sample).

\section{$\underline{\text { Analysis }}$}

All samples were analyzed using an HPLC-MS/MS method. The detector was a TSQ Quantum with electron spray ionization applied in the positive polarity mode. The collision energy for abiraterone, abiraterone-d4, abiraterone acetate and abiraterone acetate-d4 was 51, 56, 33 and $33 \mathrm{~V}$, respectively. Mass transitions for abiraterone, abiraterone-d4, abiraterone acetate and abiraterone acetate-d4 were 350.2/156.1, 354.3/160.1, 392.3/332.2 and 396.3/336.2, respectively. The following gradient of $\mathrm{MeOH} / \mathrm{H}_{2} \mathrm{O}$ was run over a Kinetex $\mathrm{C} 18$ column $(50 \times 3 \mathrm{~mm}, 1.7 \mu \mathrm{m}$; Phenomenex $)$ at a flow rate of $350 \mu \mathrm{L} / \mathrm{min}: 40 / 60(0-1 \mathrm{~min})$, changed linearly to $95 / 5$ ( 3 - $4.5 \mathrm{~min}), 40 / 60$ (4.5 - $6 \mathrm{~min})$. The injection volume was $10 \mu \mathrm{L}$ and Xcalibur was used as the software program (Thermo-Electron, San Jose, USA).

For the analysis of the mesenteric blood samples, abiraterone and abiraterone acetate were extracted from the blood before quantification by HPLC-MS/MS. After diluting $100 \mu 1$ of blood in $400 \mu \mathrm{l}$ of a mixture of acetonitrile:water (50:50), $100 \mu \mathrm{l}$ of internal standard solution (solution of abiraterone-d4 and abiraterone acetate-d4 in acetonitrile:water (50:50), $5 \mathrm{nM}$ ) and $200 \mu 1$ of $\mathrm{NaHCO}_{3}(5 \%)$ were added. After extraction with $2 \mathrm{ml}$ of methyl tert-butyl ether and centrifugation $\left(2880 \mathrm{~g}, 5 \mathrm{~min}, 4^{\circ} \mathrm{C}\right)$, the organic layer was transferred to a clean test tube and evaporated to dryness under a gentle stream of air. The residue was dissolved in $150 \mu 1$ of a solution of methanol:water (70:30 v/v), centrifuged (5 min, $20817 \mathrm{~g}, \mathrm{RT})$ and $10 \mu \mathrm{l}$ of the supernatant was injected in the HPLC-MS/MS system.

The calibration curves were linear from $500 \mathrm{nmol}$ to $0.2 \mathrm{nmol}$. Precision and accuracy errors were below $10 \%$. 


\section{$\underline{\text { Calculations }}$}

The intestinal absorptive flux of abiraterone (abiraterone acetate was not detected in rat blood) was determined from the cumulative amount of compound appearing in the mesenteric blood according to equation (1):

$$
\text { Flux }=\frac{\mathrm{dQ}}{\mathrm{dt} . \mathrm{A}}
$$

where $\mathrm{dQ} / \mathrm{dt}$ is the amount of drug appearing in the mesenteric blood over time and $\mathrm{A}$ is the surface area of the perfused cylindrical intestinal segment.

\section{$\underline{\text { Results and discussion }}$}

The purpose of this study was to gain insight into the intraluminal behavior and intestinal absorption of abiraterone acetate, an ester prodrug of the anticancer agent abiraterone. Due to its poor aqueous solubility and intestinal permeability, abiraterone acetate has been classified as BCS class IV [7]. As, according to the summary of product characteristics of ZYTIGA ${ }^{\circledR}$, the prodrug is to be taken in fasted state conditions, solubility studies, dissolution tests and absorption experiments were performed in intestinal media relevant for the fasted state. Aspirated human intestinal fluids of the fasted state (FaHIF) were used as key reference solvent systems and results were compared to those obtained with the simulated fluids of the fasted state. In view of the reported literature on prodrug instability in intestinal juices, stability of abiraterone acetate and formation of abiraterone were carefully monitored throughout the experiments.

In vivo intraluminal concentrations of abiraterone acetate and abiraterone upon intake of one tablet of $250 \mathrm{mg}$ abiraterone acetate were determined in healthy volunteers in order to evaluate the relevance of the in vitro solubility and dissolution experiments. In addition, in situ intestinal perfusion with mesenteric sampling in rats was selected as the most appropriate 
absorption model to evaluate the intestinal uptake of abiraterone and its ester prodrug from biorelevant perfusion media.

\section{$\underline{\text { Solubility measurements }}$}

Abiraterone acetate is practically insoluble in aqueous media and very slightly soluble in $0.1 \mathrm{~N}$ $\mathrm{HCl}$ [6]. Nevertheless, over the past decade, it has become abundantly clear that, in order to estimate intraluminal concentrations, solubility studies should be performed in media that are relevant for the in vivo intestinal environment [10]. In the present study, apparent solubility was determined in simulated (FaSSIF) and aspirated (FaHIF) intestinal fluids of the fasted state. The composition of the media used and their solubilizing capacity for abiraterone and abiraterone acetate are presented in Table 1.

Upon $5 \mathrm{~h}$ of incubation, the solubilities of abiraterone and abiraterone acetate in FaSSIF were $12.7 \pm 4.2$ and $64.6 \pm 5.2 \mu \mathrm{M}$, respectively. In FaHIF, the solubility of abiraterone acetate was $37.4 \pm 0.3 \mu \mathrm{M}$; however, generation of abiraterone upon incubation of the prodrug was observed, suggesting degradation of abiraterone acetate in human intestinal fluids. Interestingly, the abiraterone concentrations reached upon prodrug incubation $(23.9 \pm 0.9$ $\mu \mathrm{M}$ ), strongly exceeded the solubility values obtained when incubating abiraterone in FaHIF $(3.1 \pm 0.2 \mu \mathrm{M})$.

Due to the instable nature of the prodrug in human intestinal fluids, and the relatively late sampling time points as compared to intestinal transit times, the in vivo relevance of the apparent solubility values of abiraterone and abiraterone acetate may be limited. To further characterize the intraluminal behavior of abiraterone acetate, dissolution tests and in vivo experiments were performed. 


\section{$\underline{\text { Stability and dissolution tests }}$}

Instability in intestinal fluids has been observed for several other ester prodrugs and is generally regarded as disadvantageous to the overall prodrug approach [2,11]. A more thorough evaluation of the concentration profiles of abiraterone and abiraterone acetate upon prodrug incubation in FaHIF during the solubility studies indicates that the concentrations of abiraterone generated by prodrug hydrolysis upon 1, 2 and 5 hours of incubation were significantly higher than the solubility of abiraterone determined by incubation of abiraterone (Figure 1). Apparently, the relatively fast dissolution and subsequent degradation of abiraterone acetate in FaHIF triggers rapid and extensive formation of abiraterone. In contrast, the dissolution of abiraterone appears to be very slow as compared to dissolution of the prodrug.

To further explore the observed behavior of abiraterone acetate in FaHIF, the prodrug was incubated in dissolution vessels for a shorter time span. Powder of the prodrug or abiraterone was added to FaHIF or FaSSIF supplemented with esterases $(20 \mathrm{U} / \mathrm{ml})$ and incubated for $2 \mathrm{~h}$ with regular sampling.

Incubation of abiraterone acetate in human intestinal fluids of the fasted state confirmed the observations made during the solubility studies: prodrug incubation resulted in rapid degradation, generating abiraterone concentrations exceeding its apparent solubility. The concentration-time profile of abiraterone peaked at 20 minutes, reaching $80 \mu \mathrm{M}$, which is about fourfold higher than the apparent solubility of abiraterone determined after 24h (Figure 2a). Moreover, when comparing the in vitro behavior of the prodrug and abiraterone in FaHIF, the concentration of abiraterone generated upon prodrug incubation significantly rises above the concentrations obtained upon abiraterone incubation. At its peak concentration after 20 minutes of incubation, prodrug-induced abiraterone concentrations are more than 11-fold higher than concentrations reached upon abiraterone incubation in FaHIF. 
In contrast to its fast degradation in FaHIF, abiraterone acetate was found to be stable in FaSSIF. In an effort to increase the relevance of using FaSSIF as a solvent system to assess the intraluminal behavior of ester prodrugs, esterases $(20 \mathrm{U} / \mathrm{ml})$ were added to the simulated intestinal fluids and the $\mathrm{pH}$ was set at 7.4 , which is similar to the $\mathrm{pH}$ measured for the aspirated human intestinal fluids. In agreement with the data obtained in FaHIF, considerable formation of abiraterone was observed upon incubation of the prodrug, strongly exceeding the abiraterone concentrations measured upon abiraterone incubation (Figure 2b). As compared to the human intestinal fluids, prodrug degradation in the simulated fluids seems to be slower and abiraterone concentrations peak at 60 minutes of incubation. This is probably due to a higher hydrolytic activity in the human intestinal fluids. Nevertheless, from a qualitative point of view, the behavior of prodrug and abiraterone upon incubation in simulated and aspirated intestinal fluids is similar.

It appears that the seemingly non-productive hydrolysis of abiraterone acetate results in periods of intraluminal abiraterone supersaturation. Given the reported similar permeability of abiraterone and abiraterone acetate across Caco-2 monolayers, instability of the ester prodrug in the intraluminal environment may surprisingly be beneficial to the overall intestinal disposition of abiraterone [7].

\section{$\underline{\text { In vivo intraluminal behavior of abiraterone acetate }}$}

To estimate the in vivo relevance of the observed in vitro behavior of abiraterone acetate, intraluminal sampling was performed from the duodenum of healthy volunteers after intake of a tablet of abiraterone acetate $\left(\mathrm{ZYTIGA}^{\circledR}, 250 \mathrm{mg}\right)$. The tablet was administered together with $250 \mathrm{ml}$ of water and was taken on an empty stomach. Samples were taken over a period of $4 \mathrm{~h}$. Over the entire sampling period, only marginal concentrations of abiraterone acetate could be measured in the aspirated samples. In contrast, periods of high abiraterone concentrations 
were observed, clearly indicating that abiraterone is the major species in solution in the intraluminal environment (Figure 3a). The abiraterone concentrations observed in vivo were even higher than the concentrations that were generated during the in vitro experiments. This may be attributed to the fact that in vivo, dissolution may already occur in the stomach, in which the acidic $\mathrm{pH}$ is beneficial for the solubility of the weak base abiraterone acetate. When the total quantity of abiraterone and abiraterone acetate present in the aspirates was determined, however, significant amounts of the prodrug were detected, indicating that abiraterone acetate predominantly resides in the duodenum as undissolved powder (Figure 3b). These in vivo observations are in good agreement with the high recovery of abiraterone acetate in the faeces, which was observed in a mass balance study in healthy volunteers [8]. Moreover, as is clear from our data, the fraction of abiraterone acetate that goes into solution is rapidly converted to abiraterone. For abiraterone, the total amounts were much more in line with the solubilized fraction, indicating that, at the time of sampling, minor precipitation of abiraterone occurred at the sampling site (data not shown). This finding also suggests that supersaturation of abiraterone in vivo is more stable than in vitro, where precipitation of abiraterone was observed already after 20 min of incubation in fasted state human intestinal fluids (Figure 2a). This can be explained by the fact that in vivo, the precipitation rate might be reduced by the presence of an absorptive sink [12].

The maximum concentrations of abiraterone in the intraluminal samples were observed within the first hour after intake of the tablet, indicating a relatively fast gastrointestinal transfer. For all three volunteers, one major abiraterone peak was observed in the concentration-time profile.

Comparison of duodenal concentrations and solubility values determined for abiraterone in the aspirated intestinal samples suggests the existence of periods of intraluminal supersaturation of abiraterone. Especially at sampling times when peak concentrations were 
observed, the abiraterone concentration in solution strongly surmounted the solubility values (Figure 3c). The average abiraterone solubility in all individually aspirated samples $(18.6 \mu \mathrm{M})$ was very similar to the abiraterone solubility determined in the FaHIF pool (16.5 $\mu \mathrm{M})$, which was used in the in vitro and in situ experiments. This finding indicates that, with respect to solubility, the FaHIF pool was representative for the intraluminal samples obtained after administration of the abiraterone acetate tablet to healthy volunteers.

Overall, the in vivo observations confirm the in vitro data and indicate that the process of rapid prodrug dissolution and subsequent degradation indeed occurs in vivo and can generate intraluminal supersaturation.

\section{$\underline{\text { In situ evaluation of abiraterone absorption }}$}

Based on the observed in vitro and in vivo behavior of abiraterone acetate, the intraluminal degradation of the prodrug will be a critical factor affecting the intestinal absorption of abiraterone. To evaluate the impact of this instability on the intestinal absorption of abiraterone, in situ intestinal perfusion experiments with mesenteric blood sampling were performed in rats. Simulated and aspirated human intestinal fluids of the fasted state containing abiraterone or abiraterone acetate powder were used as perfusion media to assess the value of the prodrug approach. In all experimental conditions, an excess of powder was added to the perfusion media, and, consequently, intestinal perfusions were performed with suspensions. This was done in accordance with the presence of non-dissolved abiraterone acetate residing in the intraluminal environment, as observed in the in vivo experiments performed in this study (Figure 3b), as well as the mass balance of abiraterone, reported by Acharya et al. [8].

Upon perfusion with abiraterone suspensions in human intestinal fluids of the fasted state, low abiraterone concentrations were observed in the perfusion solution. This is in good agreement with the results obtained from the dissolution studies with abiraterone in FaHIF. 
Consequently, the abiraterone concentrations measured in the mesenteric blood were relatively low. When intestinal perfusions were performed with suspensions containing the prodrug powder, however, the high concentrations of abiraterone generated by the instability of the ester prodrug, resulted in an important increase in abiraterone flux towards the mesenteric blood (Figure 4a). Not surprisingly, no abiraterone acetate was detected in the mesenteric blood, as only a low proportion of prodrug was present in the perfusion medium, and the prodrug is also hydrolyzed in the enterocytes.

The use of simulated fluids of the fasted state in presence or absence of esterases, allows an estimation of the effect of the hydrolytic activity of the medium on the intestinal absorption of abiraterone. When perfusing abiraterone acetate suspensions in FaSSIF, only the prodrug could be measured in the perfusion medium, which was anticipated in view of the stability of the prodrug in these simulated intestinal fluids. In contrast, when using FaSSIF supplemented with esterases, both abiraterone acetate and abiraterone were present in the perfusion medium and abiraterone concentrations exceeded prodrug concentrations on average by 5 -fold during the perfusion experiments. Moreover, the cumulative concentration of abiraterone and abiraterone acetate in the perfusion solution was higher in FaSSIF supplemented with esterases as compared to plain FaSSIF. Accordingly, given the fact that abiraterone and abiraterone acetate exhibit similar permeability values, the resulting flux values of abiraterone towards the mesenteric blood when perfusing FaSSIF supplemented with esterases surmounted the abiraterone flux upon perfusion of prodrug in FaSSIF by 2-fold (Figure 4b). These findings unexpectedly indicate that adding hydrolase activity to the perfusion medium increases the intestinal absorption of abiraterone when supplemented as an acetate ester prodrug. Consistent with the observations in FaHIF, the absorptive flux values obtained upon perfusing abiraterone suspensions in simulated intestinal fluids of the fasted state were significantly lower compared to flux values obtained when perfusing the prodrug in presence 
or absence of esterases. Moreover, both perfusate concentrations of abiraterone and absorptive flux values were very similar in simulated and aspirated intestinal fluids when perfusing abiraterone suspensions.

\section{Conclusion}

The results of this study demonstrate that the success of the prodrug cannot be explained by an increased intestinal absorption and subsequent post-absorptive degradation of abiraterone acetate, which is most commonly the aim when applying a prodrug approach [11]. In contrast, based on the in vitro, in vivo and in situ results presented here, it appears that fast dissolution and subsequent immediate degradation of the prodrug is the underlying mechanism justifying the use of the prodrug over abiraterone. Based on the apparent solubility values for abiraterone in fasted state intestinal fluids, this mechanism may induce periods of intraluminal supersaturation, creating a strong driving force for intestinal absorption.

\section{$\underline{\text { Acknowledgements }}$}

This research was funded by grants from: (1) Fund for Scientific Research in Flanders (FWO), and (2) 'Onderzoeksfonds' of the KU Leuven in Belgium.

\section{$\underline{\text { References }}$}

[1] K. Masaki, M. Hashimoto, T. Imai, Intestinal first-pass metabolism via carboxylesterase in rat jejunum and ileum, Drug Metab. Dispos. 35 (2007) 1089-1095. doi:10.1124/dmd.106.013862.

[2] A.S. Borde, E.M. Karlsson, K. Andersson, K. Björhall, H. Lennernäs, B. Abrahamsson, Assessment of enzymatic prodrug stability in human, dog and simulated intestinal fluids, Eur. J. Pharm. Biopharm. 80 (2012) 630-637. doi:10.1016/j.ejpb.2011.11.011.

[3] J. Brouwers, J. Tack, P. Augustijns, In vitro behavior of a phosphate ester prodrug of amprenavir in human intestinal fluids and in the Caco-2 system: illustration of intraluminal supersaturation, Int. J. Pharm. 336 (2) (2007) 302-309.

[4] G.E. Granero, G.L. Amidon, Stability of valacyclovir: implications for its oral bioavailability, Int. J. Pharm. 317 (2006) 14-18. doi:10.1016/j.ijpharm.2006.01.050.

[5] K. Stoeckel, W. Hofheinz, J.P. Laneury, P. Duchene, S. Shedlofsky, R.A. Blouin, Stability of cephalosporin prodrug esters in human intestinal juice: implications for oral bioavailability, Antimicrob. Agents Chemother. 42 (1998) 2602-2606. 
[6] EMA (CHMP), Assessment Report For Zytiga (abiraterone). Available at http://www.ema.europa.eu/docs/en_GB/document_library/EPAR__Public_assessment_report/human/002321/WC500112860.pdf, (2011).

[7] FDA (CDER), Clinical Pharmacology and Biopharmaceutics Review(s) - Abiraterone Acetate. Available at http://www.accessdata.fda.gov/drugsatfda_docs/nda/2011/202379Orig1s000ClinPharmR .pdf, (2010).

http://www.accessdata.fda.gov/drugsatfda_docs/nda/2011/202379Orig1s000ClinPharmR .pdf (accessed September 15, 2014).

[8] M. Acharya, M. Gonzalez, G. Mannens, R. De Vries, C. Lopez, T. Griffin, et al., A phase I, open-label, single-dose, mass balance study of 14C-labeled abiraterone acetate in healthy male subjects, Xenobiotica. 43 (2013) 379-389. doi:10.3109/00498254.2012.721022.

[9] J. Bevernage, T. Forier, J. Brouwers, J. Tack, P. Annaert, P. Augustijns, Excipientmediated supersaturation stabilization in human intestinal fluids, Mol. Pharm. 8 (2011) 564-570. doi:10.1021/mp100377m.

[10] D. Hörter, J.B. Dressman, Influence of physicochemical properties on dissolution of drugs in the gastrointestinal tract, Adv. Drug Deliv. Rev. 46 (2001) 75-87.

[11] K. Beaumont, R. Webster, I. Gardner, K. Dack, Design of ester prodrugs to enhance oral absorption of poorly permeable compounds: challenges to the discovery scientist, Curr. Drug Metab. 4 (2003) 461-485.

[12] J. Bevernage, J. Brouwers, P. Annaert, P. Augustijns, Drug precipitation-permeation interplay: supersaturation in an absorptive environment, Eur. J. Pharm. Biopharm. 82 (2012) 424-428. doi:10.1016/j.ejpb.2012.07.009. 
Table 1:Individual bile salt and lecithin concentrations in FaSSIF and FaHIF and the solubility (5h) of abiraterone and abiraterone acetate in these media.

\begin{tabular}{|c|c|c|c|c|c|c|c|c|c|c|c|c|}
\hline \multirow{2}{*}{ Medium } & \multicolumn{9}{|c|}{ Bile Salt Concentration $(\boldsymbol{\mu M})$} & \multirow{2}{*}{$\begin{array}{l}\text { Lecithin concentration } \\
(\mu \mathrm{M})\end{array}$} & \multirow{2}{*}{$\begin{array}{c}\text { Abiraterone } \\
\text { solubility }(\mu \mathrm{M})\end{array}$} & \multirow{2}{*}{$\begin{array}{c}\text { Abiraterone acetate } \\
\text { solubility }(\mu \mathrm{M})\end{array}$} \\
\hline & TUDC & TCDC & TDC & GUDC & GCDC & GDC & GC & TC & Total & & & \\
\hline FaHIF & 8 & 289 & 178 & 25 & 616 & 507 & 1005 & 374 & 3002 & 986 & $3.1 \pm 0.2$ & $37.4 \pm 0.3$ \\
\hline FaSSIF & & & & & & & & 3000 & 3000 & 750 & $12.7 \pm 4.2$ & $64.6 \pm 5.2$ \\
\hline
\end{tabular}

glycocholate, TC: taurocholate 


\section{$\underline{\text { Legend for figures }}$}

Figure 1: Time dependent apparent solubility of abiraterone in FaHIF upon incubation of abiraterone (black bars) and abiraterone acetate (white bars) powder. Bars represent the mean $+\operatorname{SD}(n=3)$.

Figure 2: Concentration-time profiles of abiraterone upon dissolution of abiraterone acetate $(\square)$ and abiraterone $(\boldsymbol{\Delta})$ powder in FaHIF (2a) and FaSSIF complemented with esterases (2b). Symbols represent the mean $\pm \operatorname{SD}(n=3)$.

Figure 3: a) Duodenal concentrations of abiraterone (open symbols) and abiraterone acetate (closed symbols) over time b) Total amount (open symbols) and duodenal concentration (closed symbols) of abiraterone acetate over time. Values for the total amount are expressed as concentrations that would be measured if the total amount of abiraterone acetate were in solution in the intraluminal samples. c) Duodenal concentrations (open symbols) and solubility (closed symbols) of abiraterone over time. Symbols represent the mean $\pm \operatorname{SEM}(n=3)$.

Figure 4: Absorptive flux values of abiraterone over time in aspirated (4a) and simulated (4b) intestinal fluids of the fasted state. 4a: Squares and triangles represent absorptive flux values upon perfusions in FaHIF starting from abiraterone acetate and abiraterone suspensions, respectively. 4b: Triangles and diamonds represent absorptive flux values upon perfusions in FaSSIF starting from abiraterone and abiraterone acetate suspensions, respectively. Squares represent absorptive flux values upon perfusions in FaSSIF supplemented with esterases starting from abiraterone acetate suspensions. Symbols represent the mean $\pm S D(n=3)$. 


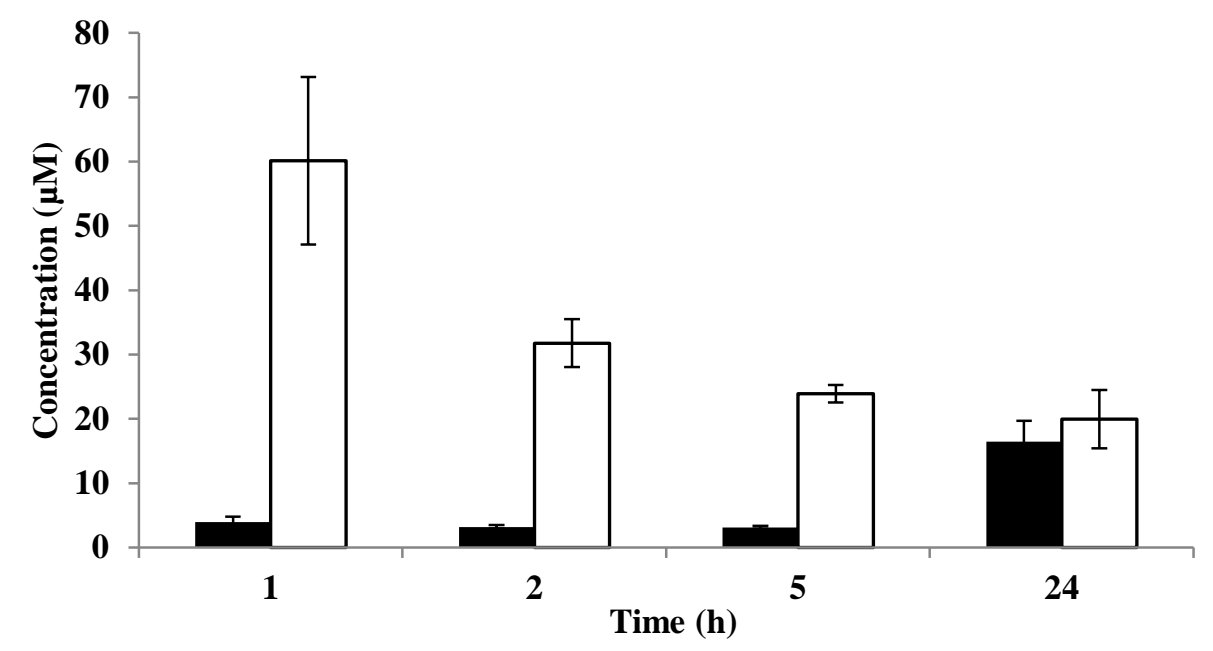

a abiraterone incubation $\square$ abiraterone acetate incubation

Figure 1
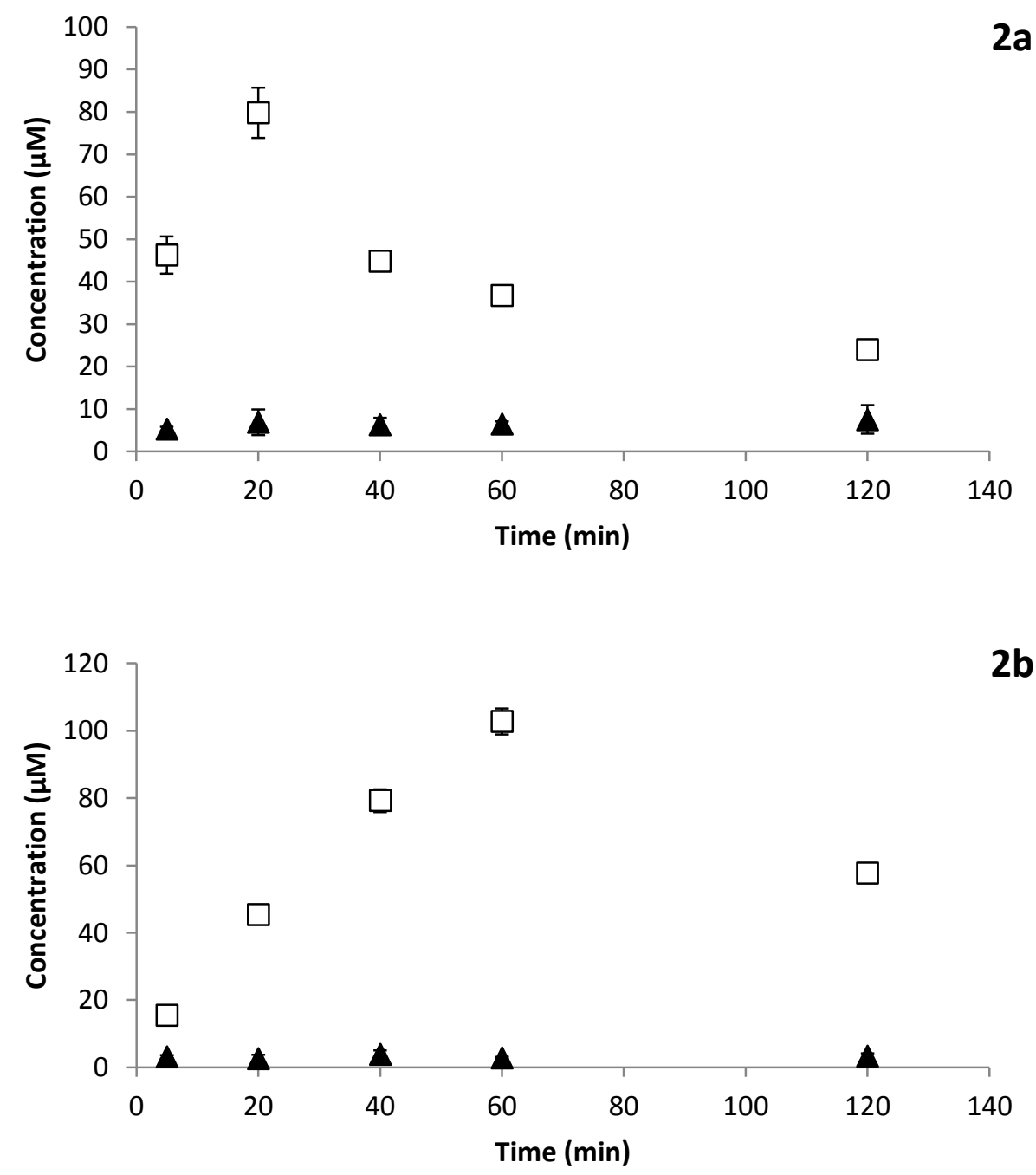

Figure 2 


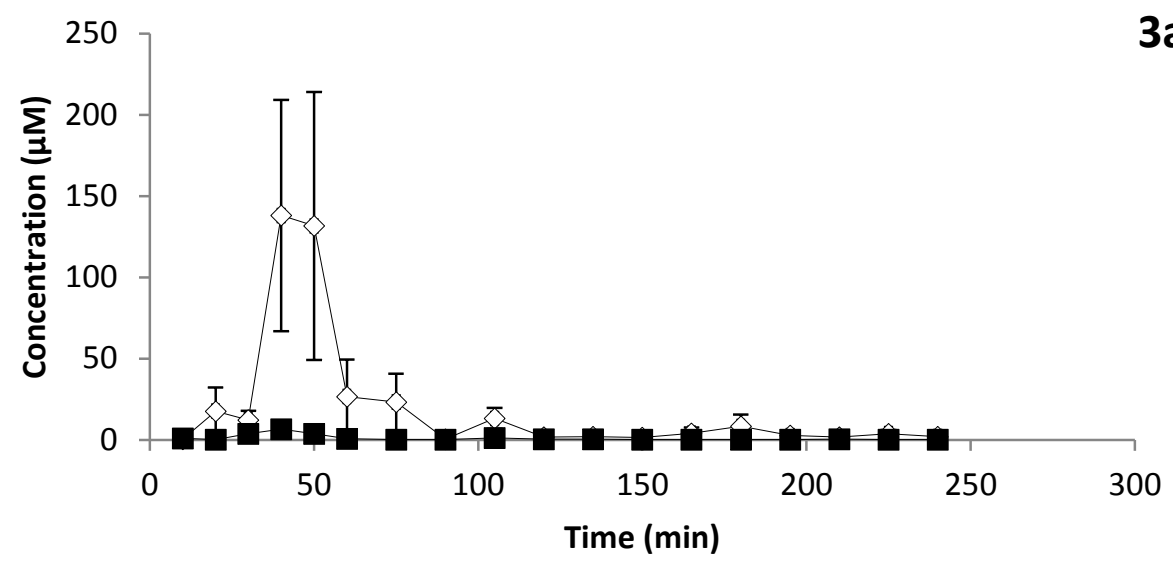

$3 a$

$\diamond A-A A$

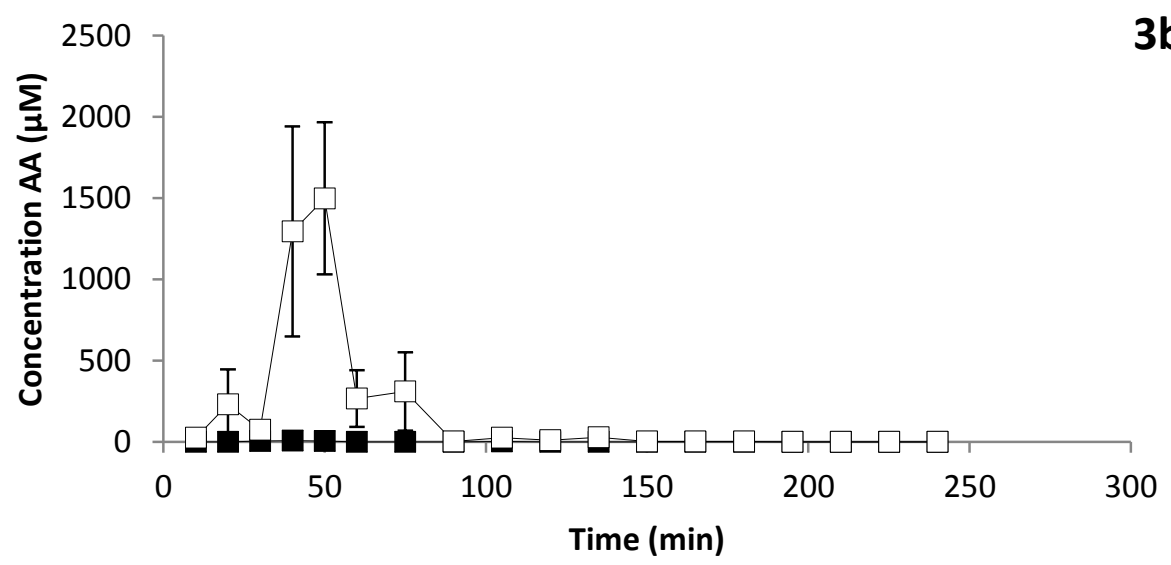

- in solution $\quad-\square-$ total

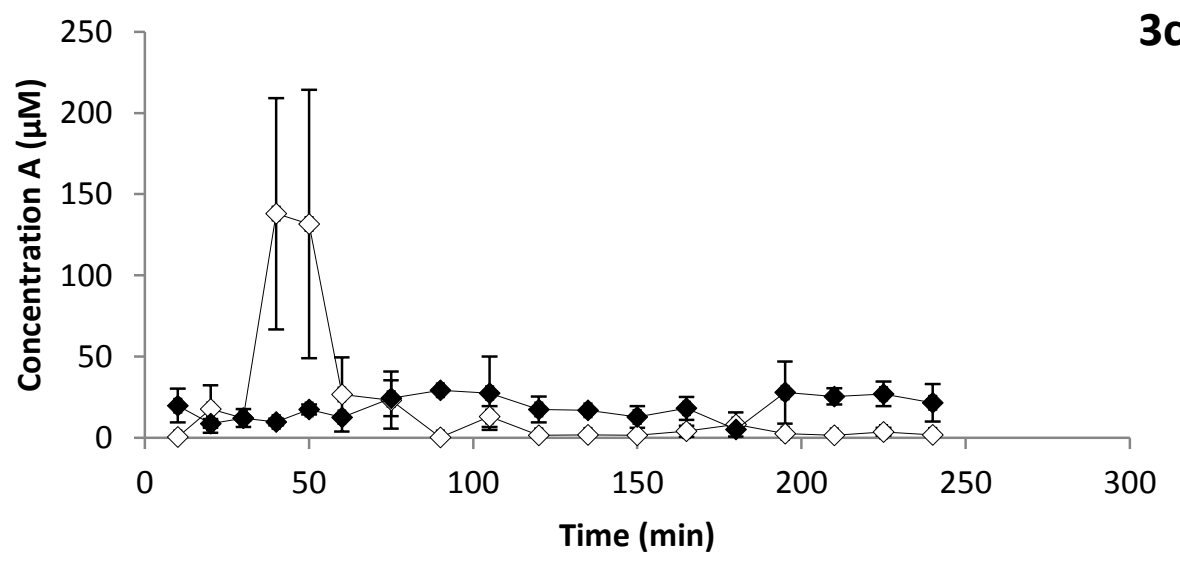

$\checkmark$ in solution $\prec$ solubility

Figure 3 


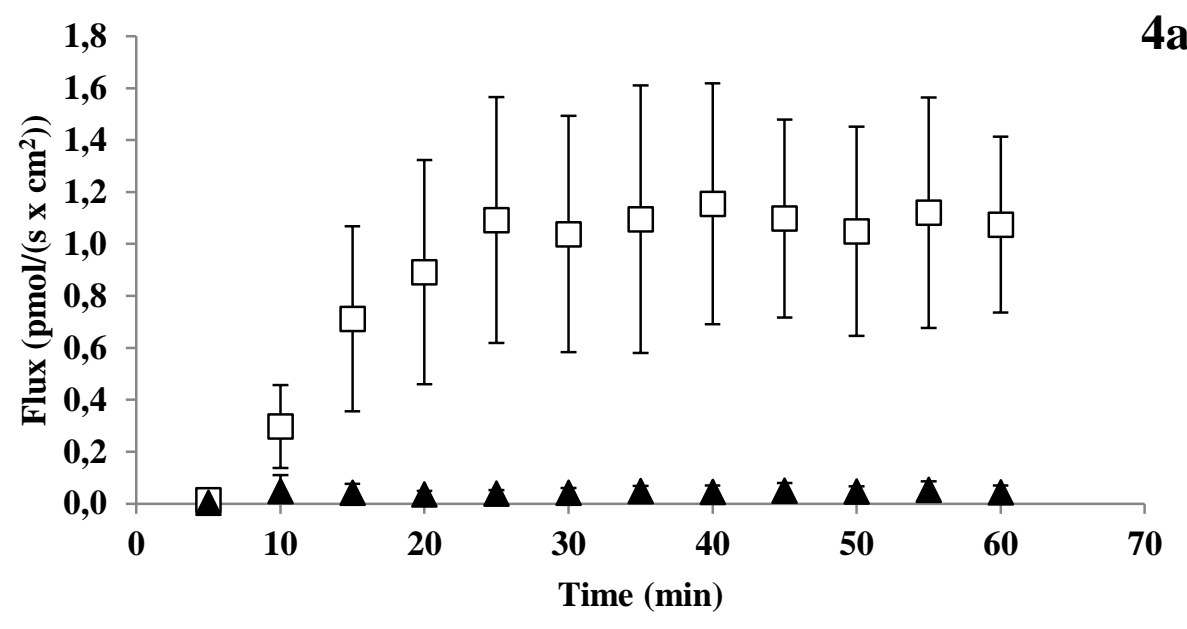

$\square \mathrm{AA}$ in FaHIF $\triangle \mathrm{A}$ in FaHIF

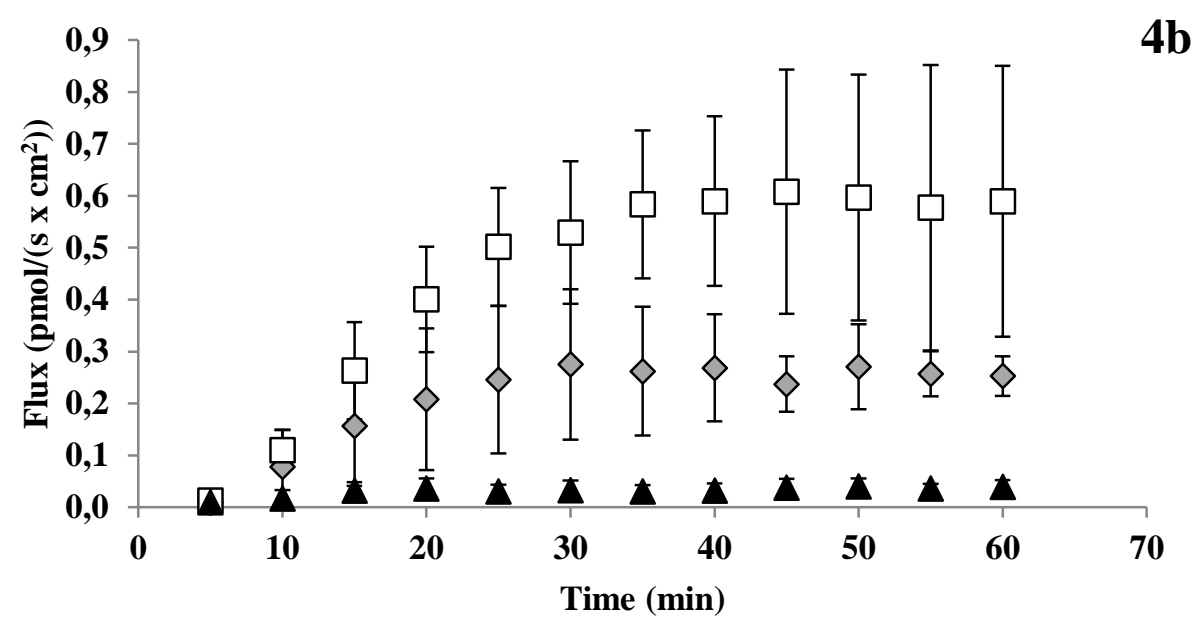

$\diamond \mathrm{AA}$ in FaSSIF $\square \mathrm{AA}$ in FaSSIF + esterase $\triangle \mathrm{A}$ in FaSSIF

Figure 4 